\title{
Emission lifetime study of fluorescence probes based on G-quadruplex oligonucleotides end-labeled with pyrene moieties
}

\author{
A. Dembska ${ }^{\mathrm{a}}$, T. Pedzinski ${ }^{\mathrm{a}}, \mathrm{S}$. Takenaka ${ }^{\mathrm{b}}$ and B. Juskowiak ${ }^{\mathrm{a}, *}$ \\ ${ }^{a}$ Faculty of Chemistry, A. Mickiewicz University, Poznan, Poland \\ ${ }^{\mathrm{b}}$ Department of Applied Chemistry, Kyushu Institute of Technology, Kitakyushu, Japan
}

\begin{abstract}
Fluorescence lifetime study of two probes abbreviated as Py-Htelom-Py and Py-TBA-Py, carrying pyrene moieties at both termini and sequences of Human telomere and Thrombin Binding Aptamer, respectively are reported. The effect of potassium ion on the photophysical processes was examined in order to elucidate factors that facilitate the production of excimer emission. Emission kinetics data indicated that the relative orientation of pyrene and neighboring nucleobase (guanine, adenine, thymine) plays a crucial role in determining both the rate of electron-transfer quenching of pyrene excited state and the efficiency of excimer emission.
\end{abstract}

Keywords: Excimer, fluorescence, G-quadruplex, lifetime, potassium probe

\section{Introduction}

Certain DNA sequences that are guanine-rich can form four-stranded structures called G-quadruplexes under specific cation conditions. Typically, three or four G-quartets are stacked and held together by $\pi-\pi$ nonbonded attractive interactions, but formed G-quadruplexes may have different topological structures depending on the oligonucleotide sequence and environmental conditions [5]. Recently, we have developed fluorescent oligonucleotide probes based on the G-quadruplex scaffold for detecting potassium (PSO - Potassium Sensing Oligonucleotide) $[1,2,4,8]$. The approach that exploited excimer emission of the pyrene labels $[1,4]$ is of particular interest since it enables an insight into label-label and label/nucleobases interaction processes. Moreover, due to the long lifetime of the pyrene label [10], such probes can be useful for time-resolved fluorescence monitoring of potassium in biological samples since pyrene excimer signal can be separated from biological background interference.

Here, we report on the fluorescence lifetime study of two fluorescent probes shown in Scheme 1, abbreviated as Py-TBA-Py and Py-Htelom-Py. They carry pyrene moieties at both termini of a $\mathrm{d}\left(\mathrm{G}_{2} \mathrm{~T}_{2} \mathrm{G}_{2} \mathrm{TGTG}_{2} \mathrm{~T}_{2} \mathrm{G}_{2}\right)$ sequence of Thrombin Binding Aptamer (TBA) and a d $\left(\mathrm{AG}_{3}\left(\mathrm{~T}_{2} \mathrm{AG}_{3}\right)_{3} \mathrm{~A}\right)$ sequence of Human telomere (Htelom). The effect of potassium on the photophysical processes was examined in order to elucidate factors that may facilitate the production of excimer emission.

\footnotetext{
*Corresponding author: Prof. Bernard Juskowiak, A. Mickiewicz University, Department of Chemistry, ul. Grunwaldzka 6, 60-780 Poznan, Poland. Tel.: +48 618291 467; Fax: +48 618291 505; E-mail: juskowia@ amu.edu.pl.
} 
<smiles>O=C(Cc1ccc2cccc3c4c(c5c(cccc15)C=CC=4)=c23)NCCCCCCCNC(=O)Cc1ccc2ccc3cccc4ccc1c2c34</smiles>

Py-Htelom-Py<smiles>O=C(Cc1ccc2ccc3cccc4ccc1c2c34)NCCCCCCOP(=O)(O)OCc1ccccc1</smiles><smiles>O=C(Cc1ccc2ccc3cccc4ccc1c2c34)NCCCCCC(CO)OP(=O)(O)O</smiles>

Py-TBA-Py

Scheme 1.

\section{Experimental}

\subsection{Materials}

Pyrene-labeled oligonucleotides (Scheme 1) were custom-synthesized by Sigma-Genosys Japan (Ishikari, Japan) and purified by reversed phase HPLC and their identities were confirmed by MALDITOF MS (Voyager ${ }^{\mathrm{TM}}$ ). Stock solutions of the oligonucleotides were prepared in ultrapure water (Polwater, Poland). Other chemicals were of analytical grade. Sample solutions containing $1 \mu \mathrm{M}$ oligonucleotide probe, $5 \mathrm{mM}$ Tris- $\mathrm{HCl}$ buffer ( $\mathrm{pH} 7.4$ ) and required metal cation were heated at $90^{\circ} \mathrm{C}$ for $5 \mathrm{~min}$ and then incubated overnight at $4^{\circ} \mathrm{C}$ before measurements.

\subsection{Fluorescence lifetime measurements}

Fluorescence decays were obtained using a TCSPC (Time Correlated Single Photon Counting) method. Measurements were made with an IBH Consultants (Glasgow, Scotland) model 5000 fluorescence lifetime spectrometer equipped with the NanoLED type diode $\left(\lambda_{\mathrm{exc}}=340 \mathrm{~nm}\right.$, fwhm $\left.=800 \mathrm{ps}\right)$ as an excitation source. The standard procedure for performing these measurements included taking an instrument profile and counting photon signals until at least 5000 counts were collected at the maxima of the fluorescence decay histograms. Reconvolution of fluorescence decay curves was performed using the IBH Consultants Version 4 software. The quality of the fits was judged from the chi-square values $\left(\chi^{2} \leqslant 1.5\right)$ and the random distribution of weighted residuals. The fluorescence intensity was monitored at two wavelengths, 390 and $480 \mathrm{~nm}$ characteristic of pyrene monomer and excimer emission, respectively. Solvents were checked under the conditions used in the fluorescence decay measurements, and they were found to exhibit emission small enough to be negligible relative to their influence on the lifetimes measured.

\section{Results and discussion}

\subsection{Fluorescence properties of probes}

Steady-state fluorescence study of the Htelom and TBA probes revealed remarkable differences in their spectral characteristics as reported previously $[1,4]$. The Py-TBA-Py probe gave efficient excimer emission in the presence of potassium [4] but the sensor with the human telomeric sequence showed only 
quenching of the pyrene monomer emission without noticeable excimer contribution [1]. The absence of excimer emission for Py-Htelom-Py probe was not due to the lack of quadruplex formation since attachment of pyrene moieties stabilizes quadruplex structure as it was evidenced by CD measurements [1]. Therefore, the attached oligonucleotide may have relaxed the pyrene excited state sufficiently fast that was not enough time for excimer formation. Alternatively, the nucleobases could have quenched the excimers that were produced. Irrespective which mechanism is responsible for the lack of excimer emission in case of Py-Htelom-Py, the different spectral characteristics of both probes must be related to the topological differences in their folded structures (quadruplexes). The fluorescence lifetime study should provide additional evidence that particular quadruplex topology can facilitate excimer emission or not.

\subsection{Lifetime study}

The excited state kinetics was monitored at $390 \mathrm{~nm}$ (pyrene monomer fluorescence) and at $480 \mathrm{~nm}$ characteristic of excimer emission. Decay experiments were also carried out with a buffer and unmodified oligonucleotide solutions to estimate the importance of background fluorescence. In both cases a weak emission was observed that originated from unknown impurities. This background emission was shorter than $1 \mathrm{~ns}$, significantly lower than emission arising from the pyrene label and was neglected in further study. On the contrary, the free pyrene label possesses large emission quantum yield and long lifetime, which are dependent on the media conditions (solvent, quenchers). The Tris- $\mathrm{HCl}$ buffer used in our experiments was not deoxygenated, therefore the lifetime for 1-pyrenebutanoic acid (PBA) of $\sim 85 \mathrm{~ns}$, shown in Table 1, may differ from reported elsewhere. For example, Zahavy et al. reported lifetime of $180 \mathrm{~ns}$ for PBA in deaerated phosphate buffer [12] but Telser et al. obtained $83 \mathrm{~ns}$ in aerated phosphate buffer containing $100 \mathrm{mM} \mathrm{NaCl}$ [7]. Pyrenebutanoic acid showed mono-exponential emission decay contrary to pyrene labeled probes, Py-TBA-Py and Py-Htelom-Py, for which a tri-exponential $F(t)=A+B_{1} \exp \left(-t / \tau_{1}\right)+B_{2} \exp \left(-t / \tau_{2}\right)+B_{3} \exp \left(-t / \tau_{3}\right)$ was needed to fit the data for both, free and potassium-complexed probes. The relative amplitudes (fractions of the emitting species, $\alpha_{i}$ ) were calculated using $\alpha_{i}=B_{i} / \sum_{i} B_{i}$ and average lifetimes $\langle\tau\rangle$, according to $\langle\tau\rangle=\sum_{i} \alpha_{i} \tau_{i}$. Examples of experimental decays for Py-TBA-Py probe in the absence and the presence of $100 \mathrm{mM} \mathrm{KCl}$ are shown in Fig. 1. A significant difference between decays for free and potassium-bound probes can be seen. Signal monitored at the monomer emission $(\lambda=390 \mathrm{~nm})$ decays faster after addition of potassium ion (Fig. 1B), whereas emission recorded at $480 \mathrm{~nm}$ decays with much longer average lifetimes (Fig. 1A) indicating an appearance of excimer emission. Decays for Py-Htelom-Py systems exhibited similar trends,

Table 1

Average lifetimes $(\langle\tau\rangle)$ for the quadruplexes of Py-Htelom-Py and Py-TBA-Py with potassiumion

\begin{tabular}{llcr}
\hline Probe & Conditions & \multicolumn{2}{c}{ Average lifetime $\langle\tau\rangle(\mathrm{ns})$} \\
\cline { 3 - 4 } & & $390 \mathrm{~nm}$ & $480 \mathrm{~nm}$ \\
\hline PBA & No salt & 85.4 & - \\
Py-TBA-Py & No salt & 6.2 & 4.3 \\
& $100 \mathrm{mM} \mathrm{KCl}$ & 2.9 & 45.0 \\
Py-Htelom-Py & No salt & 11.0 & 9.3 \\
& $100 \mathrm{mM} \mathrm{KCl}$ & 6.3 & 3.4 \\
\hline
\end{tabular}

Note: The relative error for lifetime determination was within $\pm 10-30 \%$ with results for shortest lifetimes to be more uncertain. 
(A)

(B)

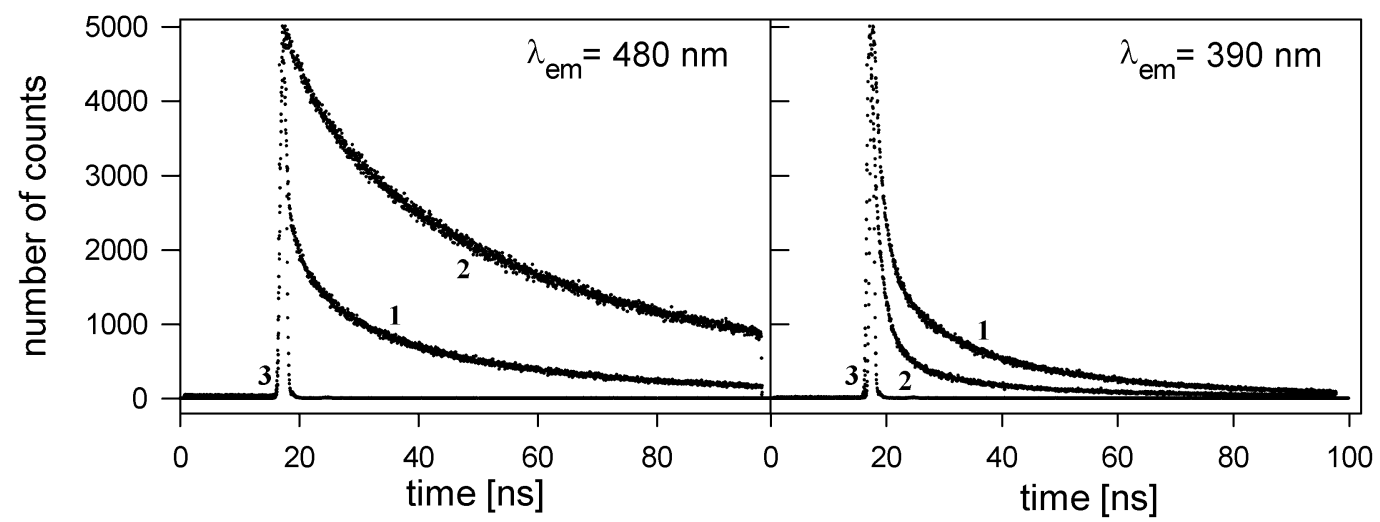

Fig. 1. Emission decay curves of Py-TBA-Py probe $(1 \mu \mathrm{M})$ in Tris- $\mathrm{HCl}$ monitored at $390 \mathrm{~nm}(\mathrm{~A})$ and $480 \mathrm{~nm}(\mathrm{~B})$ in the absence of metal cation (1) and in the presence of $100 \mathrm{mM} \mathrm{KCl}(2)$. The excitation was at $340 \mathrm{~nm}$ and trace 3 represents the lamp profile.

irrespective which wavelength was chosen for decay monitoring (data not shown). This suggested the lack of additional long wavelength emission in good agreement with their steady-state spectra [1]. Figure 2 shows examples of lifetime distribution for free probes and their potassium quadruplexes. It is striking that more that $70 \%$ of the pyrene excited states in the labeled oligonucleotides have an emission component with lifetime about $1 \mathrm{~ns}$ or even below (Fig. 2). The medium lifetime component is in the range 7.5-8.5 ns (18-15\%) for both free probes, while long-lived species have lifetimes of $33(11 \%)$ and $60 \mathrm{~ns}(15 \%)$ for TBA and Htelom-based probes, respectively. Formation of quadruplexes in the presence of potassium cation changes the lifetime distributions in both investigated systems. The most pronounced effects can be observed for Py-TBA-Py/KCl quadruplexes (Fig. 2A, B). The population of the long-lived component of monomer emission with lifetime of $\sim 30 \mathrm{~ns}(11 \%)$ decreased significantly $(3 \%)$ in the presence of potassium ion without particular shift in lifetime (Fig. 2A). On the contrary, fraction of the short-lived species with lifetime of $\sim 1 \mathrm{~ns}(71 \%)$ increased markedly (85\%). As reported already, the Py-TBA-Py probe in the presence of potassium showed fluorescence quenching of monomer band $(390 \mathrm{~nm})$ and large fluorescence enhancement in long wavelength range $(480 \mathrm{~nm})$, which was attributed to the excimer emission [4]. Indeed, lifetime distribution of the Py-TBA-Py potassium quadruplex provides solid proof that supports formation of excimers in the system. The long-lived component of $\sim 30 \mathrm{~ns}(10 \%)$ was shifted to $\sim 80 \mathrm{~ns}$ with remarkable increase in population (38\%). The remaining two lifetimes $(\sim 1$ and $\sim 7 \mathrm{~ns}$ ) also were shifted to higher values of $\sim 6$ and $27 \mathrm{~ns}$, respectively. As the three lifetimes characterize the emission decays of investigated systems it is not necessarily true that there are only three distinct deactivation processes of pyrene excited state and that the particular lifetime $\tau_{i}$ denotes the same emitting component in different systems. We do not intend to propose a microscopic description of the system; the lifetime data presented here should be rather regarded as a qualitative measure of the pyrene-pyrene and pyrene-nucleobases interactions. Thus one may conclude, that PyTBA-Py/potassium quadruplex formation leads to the efficient label-label interactions that results in redistribution of the lifetimes of monomer excited state (shortening of the average lifetime) accompanied by the appearance of excimer emission with much slower deactivation processes. The chair-type structure of the TBA quadruplex shown in Fig. 3A with the face-to-face arrangement of pyrene labels is undoubtedly responsible for the generation of excimer emission $[4,9]$. 

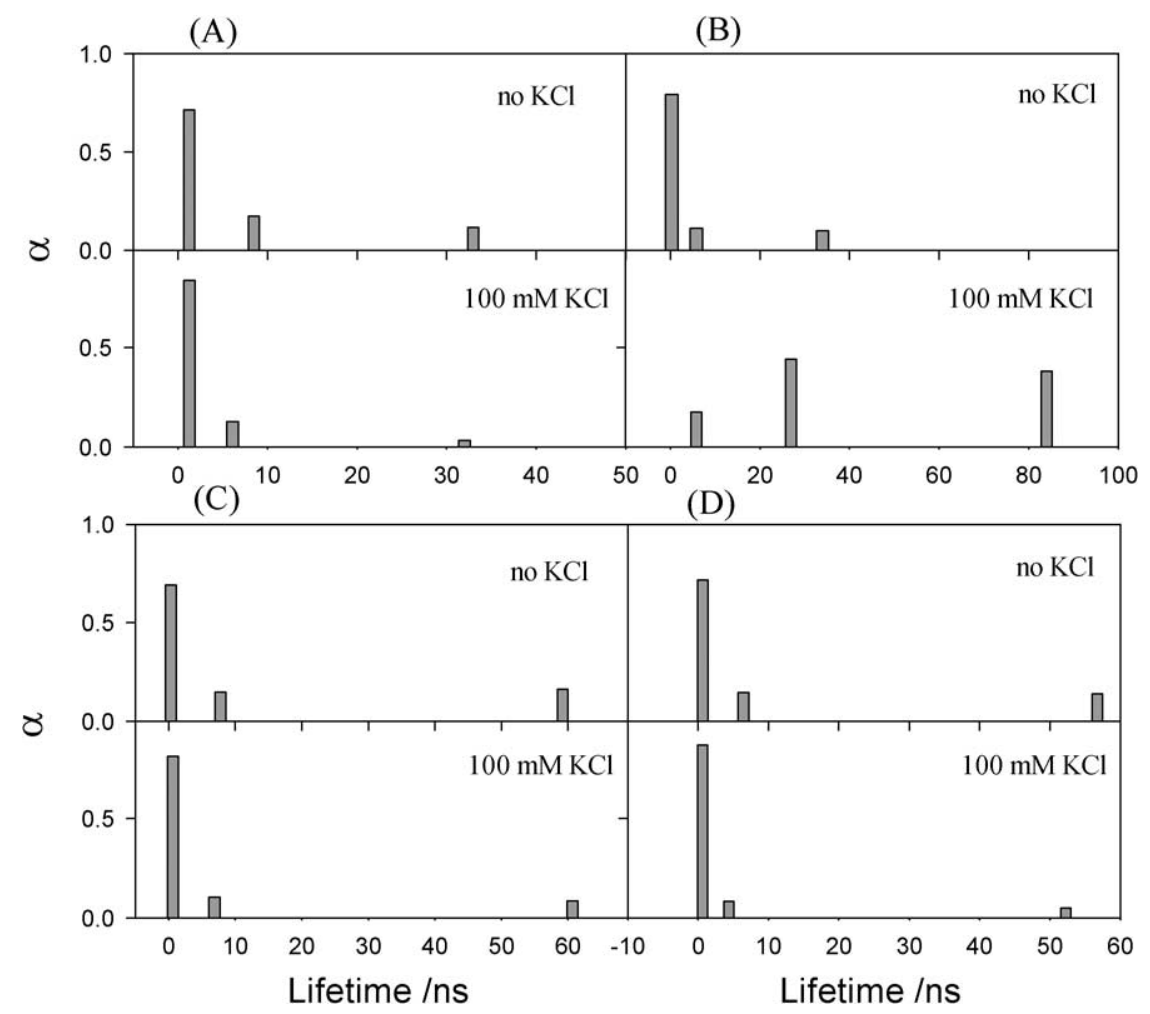

Fig. 2. Lifetime distribution for Py-TBA-Py (A, B) and Py-Htelom-Py (C, D) monitored at $390 \mathrm{~nm}$ (A, C) and $480 \mathrm{~nm}$ (B, D). Each panel shows results for free probe (top) and for potassium quadruplex (bottom).

The Htelom-based probe exhibited different decays in the presence of potassium. Inspection of lifetime distribution shown in Fig. 2C, D reveals that similar lifetime data were obtained for both wavelengths of monitoring, in good agreement with the lack of excimer emission band in the fluorescence spectra. Results calculated for $390 \mathrm{~nm}$ seem to be more reliable due to higher signal-to-noise ratio at this wavelength. Interestingly, free Py-Htelom-Py probe has the long-lived lifetime of $\sim 60 \mathrm{~ns}$, which is almost two-times longer than that for TBA probe but still remarkable shorter than obtained for PBA. Addition of potassium has modest effect on the values of lifetimes but affected significantly their relative distribution. The population of the species with shortest lifetime increased from $69 \%$ to $82 \%$, the fractions of medium and longest lifetime components decreased from $15 \%$ to $10 \%$ and from $16 \%$ to $8 \%$, respectively. Efficient deactivation kinetics of the excited state of Py-Htelom-Py/potassium probe is in good agreement with strong fluorescence quenching observed in this system [1], but the nature of the quenching seems to be static in origin since no lifetime shortening is observed. One can assume that nonfluorescent pyrene dimers and/or pyrene/nucleobase stacking complexes can be responsible for observed lifetime distribution. Formation of nonfluorescent pyrene dimers should be rather ruled out since they were not observed in similar TBA system. Explanation of the feasibility of the pyrene/nucleobase stacking interactions should involve differences in topologies of particular quadruplexes. TBA oligonucleotide forms chair-type quadruplexes [5], and as it is shown in Fig. 3A this quadruplex architecture prefers pyrene-pyrene interactions to pyrene-guanine quartet stacking. Oligonucleotides with human telomere sequence are known to form quadruplexes that exhibit structural polymorphism in presence of specific metal cations $[6,9,11]$. Formation of the hybrid- and/or the propeller-type quadruplex struc- 


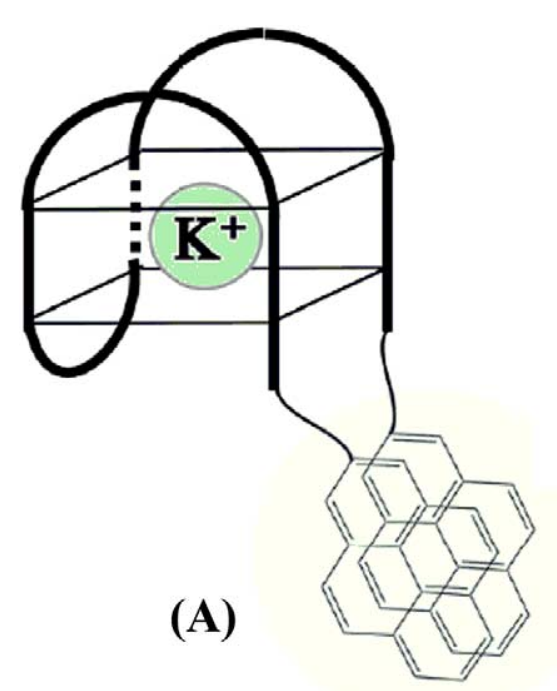

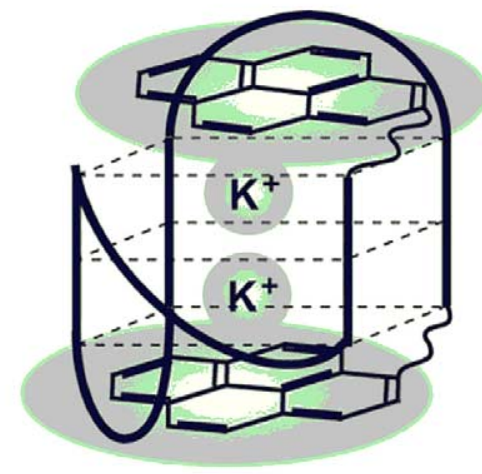

(B)

Fig. 3. Expected tetraplex structures and arrangement of pyrene labels for Py-TBA-Py/K ${ }^{+}$(A) and Py-Htelom-Py/K ${ }^{+}$(B) quadruplexes.

tures can explain observed redistribution of lifetime population in the presence of $\mathrm{KCl}$. It was shown recently that hybrid-type quadruplex dominates in $\mathrm{KCl}$ solution while propeller-type quadruplex was reported for solid state samples [6,11]. Both these quadruplexes possess external guanine tetrads exposed to hydrophobic planar guest molecules. Pyrene moiety can thus effectively stack on the guanine tetrads facilitating static or electron transfer quenching. It is generally accepted that anchoring the pyrene moiety to DNA oligonucleotide probe results in substantial decrease in fluorescence quantum yield, which is ascribed to the electron transfer (ET) between pyrene and DNA nucleobases (except for adenine [3]). Thymine bases present in lateral loops of hybrid-type quadruplex can quench additionally the emission of pyrene. Figure 3B shows the model of a hybrid-type quadruplex of Py-Htelom-Py stabilized by stacking interactions of pyrene labels.

\section{Conclusions}

Results of the fluorescence lifetime measurements of potassium G-quadruplex formation by the pyrene-modified oligonucleotides with sequences of thrombin binding aptamer (Py-TBA-Py) and human telomeric sequence (Py-Htelom-Py) were presented. All decays of investigated systems could be characterized by a tri-exponential rate. Calculated lifetimes and their fractional distribution depended on the sequence of attached oligonucleotide and reflected the extent of quenching of pyrene excited state upon interaction with nucleobases. On the other hand, structure of quadruplex affects seriously label-label and label-nucleobase interactions. Quadruplexes of the Py-Htelom-Py probe that form with potassium ion a hybrid and/or a propeller-type structures, facilitate deactivation of the excited state of pyrene due to stacking interactions between pyrene and guanine tetrads. The quadruplex topology that enables an efficient label-label interactions thus generation of excimer emission with very long lifetime of $\sim 80 \mathrm{~ns}$ appeared to be a chair-type structure formed by Py-TBA-Py probe, in the presence of potassium ion. 


\section{References}

[1] H. Hayashida, J. Paczesny, B. Juskowiak and S. Takenaka, Bioorg. Med. Chem. 16 (2008), 9871-9881.

[2] B. Juskowiak, E. Galezowska, A. Zawadzka, A. Gluszynska and S. Takenaka, Spectrochim. Acta A 64 (2006), $835-843$.

[3] M. Manoharan, K.L. Tivel, M. Zhao, K. Nafisi and T.L. Netzel, J. Phys. Chem. B 99 (1995), 17461-17472.

[4] S. Nagatoishi, T. Nojima, B. Juskowiak and S. Takenaka, Angew. Chem. Int. Ed. 44 (2005), 5067-5070.

[5] S. Neidle and S. Balasubramanian (eds), Quadruplex Nucleic Acids, RSC Biomolecular Sciences, Cambridge, 2006.

[6] G.N. Parkinson, M.P. Lee and S. Neidle, Nature 417 (2002), 876-880.

[7] J. Telser, K.A. Cruickshank, L.E. Morrison, T.L. Netzel and C.K. Chan, J. Am. Chem. Soc. 111 (1989), 7226-7232.

[8] H. Ueyama, M. Takagi and S. Takenaka, J. Am. Chem. Soc. 124 (2002), 14286-14287.

[9] Y. Wang and D.J. Patel, Structure 1 (1993), 263-282.

[10] F.M. Winnik, Chem. Rev. 93 (1993), 587-614.

[11] Y. Xu, Y. Noguchi and H. Sugiyama, Bioorg. Med. Chem. 14 (2006), 5584-5591.

[12] E. Zahavy and M.A. Fox, J. Phys. Chem. B 103 (1999), 9321-9327. 


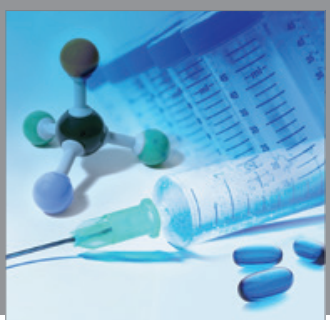

International Journal of

Medicinal Chemistry

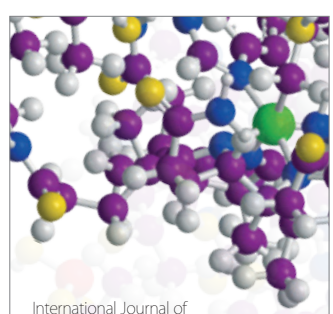

Carbohydrate Chemistry

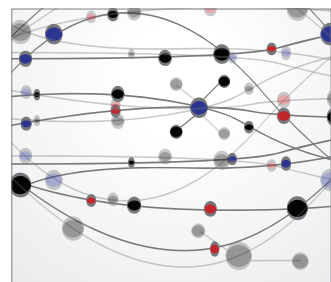

The Scientific World Journal
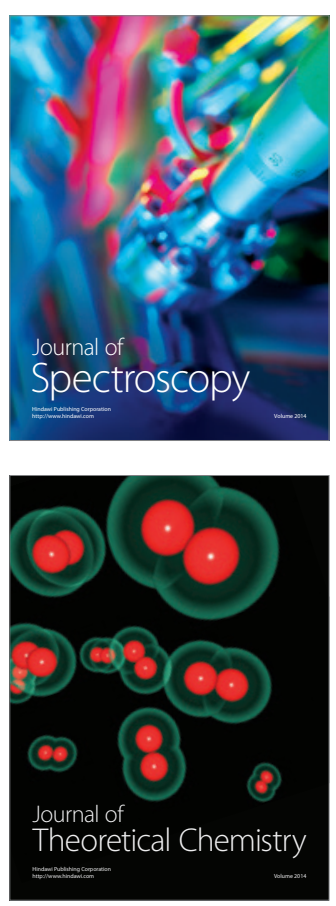
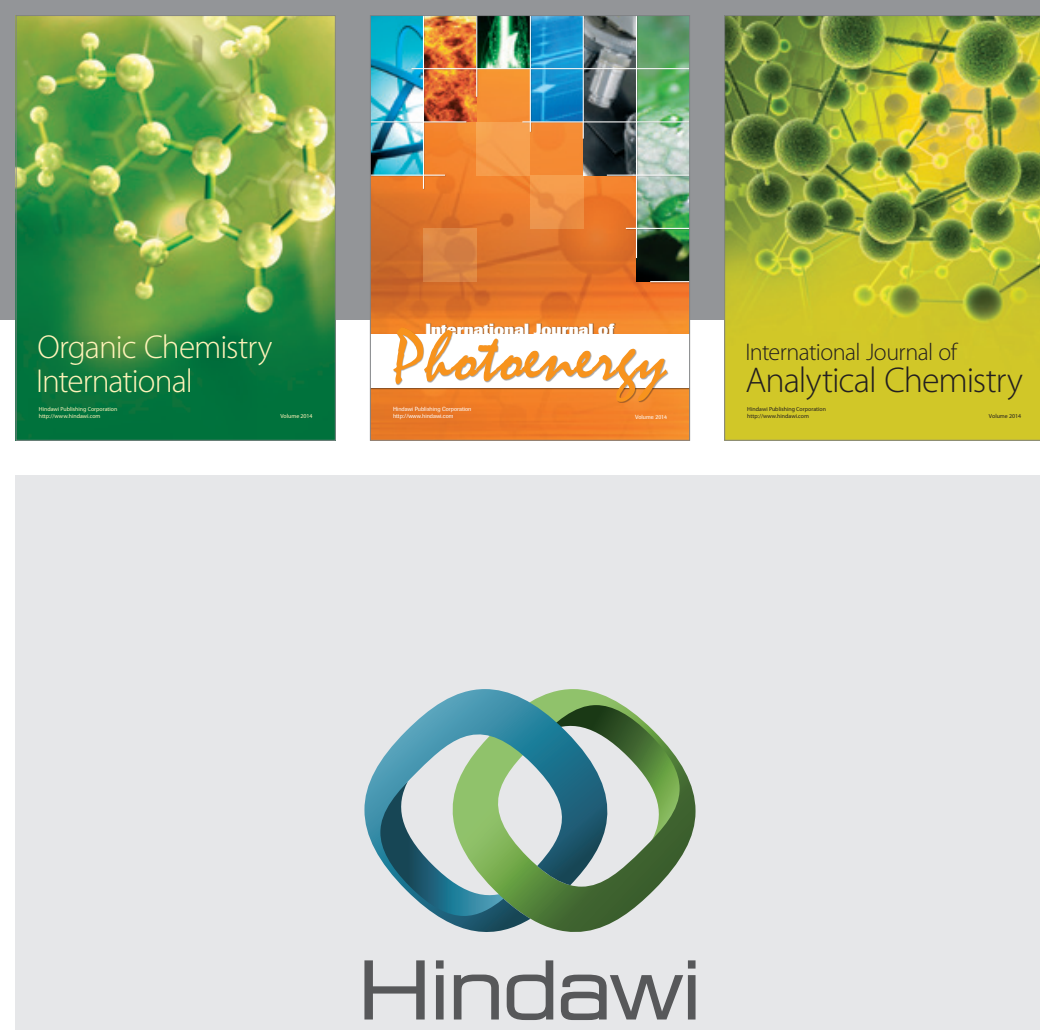

Submit your manuscripts at

http://www.hindawi.com
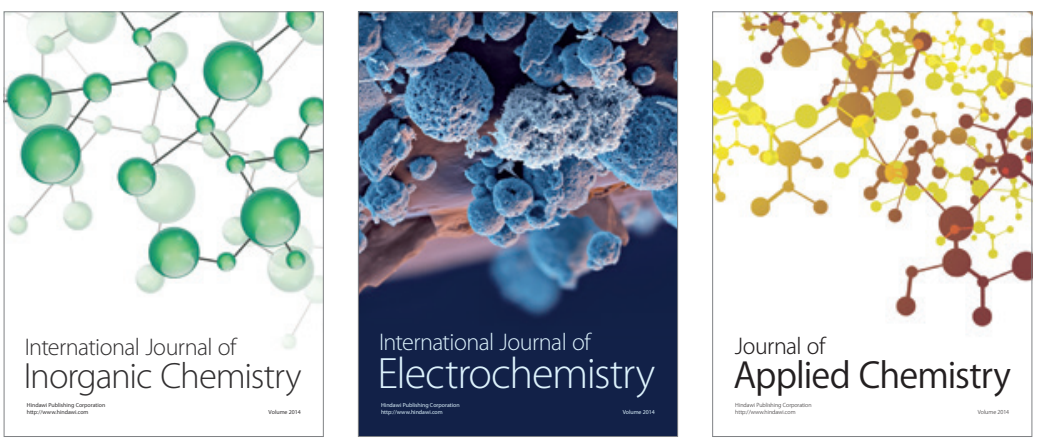

Journal of

Applied Chemistry
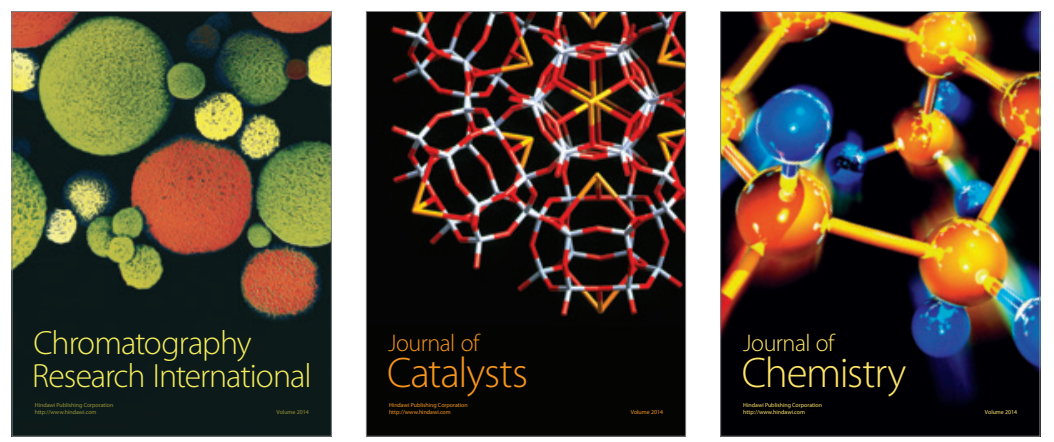
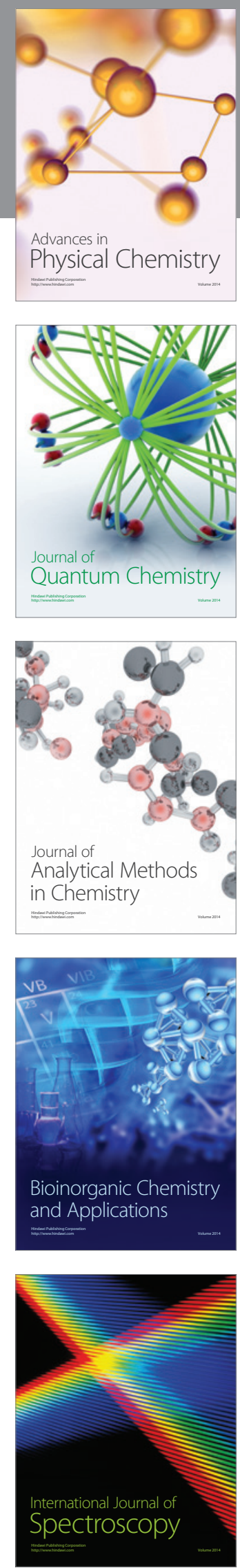